\title{
ON LIMITING RELATIONS FOR CAPACITIES
}

\author{
V.I. KOLYADA
}

\begin{abstract}
The paper is devoted to the study of limiting behaviour of Besov capacities $\operatorname{cap}\left(E ; B_{p, q}^{\alpha}\right)(0<\alpha<1)$ of sets in $\mathbb{R}^{n}$ as $\alpha \rightarrow 1$ or $\alpha \rightarrow 0$. Namely, let $E \subset \mathbb{R}^{n}$ and

$$
J_{p, q}(\alpha, E)=[\alpha(1-\alpha) q]^{p / q} \operatorname{cap}\left(E ; B_{p, q}^{\alpha}\right) .
$$

It is proved that if $1 \leq p<n, 1 \leq q<\infty$, and the set $E$ is open, then $J_{p, q}(\alpha, E)$ tends to the Sobolev capacity $\operatorname{cap}\left(E ; W_{p}^{1}\right)$ as $\alpha \rightarrow 1$. This statement fails to hold for compact sets. Further, it is proved that if the set $E$ is compact and $1 \leq p, q<\infty$, then $J_{p, q}(\alpha, E)$ tends to $2 n^{p}|E|$ as $\alpha \rightarrow 0(|E|$ is the measure of $E)$. For open sets it is not true.
\end{abstract}

\section{INTRODUCTION}

The Sobolev space $W_{p}^{1}\left(\mathbb{R}^{n}\right)(1 \leq p<\infty)$ is defined as the class of all functions $f \in L^{p}\left(\mathbb{R}^{n}\right)$ for which all first-order weak derivatives $\partial f / \partial x_{k}=D_{k} f(k=1, \ldots, n)$ exist and belong to $L^{p}\left(\mathbb{R}^{n}\right)$.

The classical embedding theorem with limiting exponent states that if $1 \leq p<n$, then for any $f \in W_{p}^{1}\left(\mathbb{R}^{n}\right)$

$$
\|f\|_{p^{*}} \leq c \sum_{k=1}^{n}\left\|D_{k} f\right\|_{p}, \quad \text { where } \quad p^{*}=\frac{n p}{n-p} .
$$

This theorem was proved by Sobolev in 1938 for $1<p<n$ and by Gagliardo and Nirenberg in 1958 for $p=1$ (see [24, Chapter 5]).

Embeddings with limiting exponent are also true for some spaces defined in terms of moduli of continuity.

Let $f \in L^{p}\left(\mathbb{R}^{n}\right)(1 \leq p<\infty)$ and $k \in\{1, \ldots, n\}$. The partial modulus of continuity of $f$ in $L^{p}$ with respect to $x_{k}$ is defined by

$$
\omega_{k}(f ; \delta)_{p}=\sup _{0 \leq h \leq \delta}\left(\int_{\mathbb{R}^{n}}\left|f\left(x+h e_{k}\right)-f(x)\right|^{p} d x\right)^{1 / p}
$$

( $e_{k}$ is the $k$ th unit coordinate vector).

2010 Mathematics Subject Classification. Primary 46E35; Secondary 31B15.

Key words and phrases. Capacity; Moduli of continuity; Sobolev spaces; Besov spaces. 
Let $0<\alpha<1,1 \leq p<\infty, 1 \leq q \leq \infty$, and $k \in\{1, \ldots, n\}$. The Nikol'skiü-Besov space $B_{p, q ; k}^{\alpha}\left(\mathbb{R}^{n}\right)$ consists of all functions $f \in L^{p}\left(\mathbb{R}^{n}\right)$ such that

$$
\|f\|_{b_{p, q ; k}^{\alpha}} \equiv\left(\int_{0}^{\infty}\left(t^{-\alpha} \omega_{k}(f ; t)_{p}\right)^{q} \frac{d t}{t}\right)^{1 / q}<\infty
$$

if $q<\infty$, and

$$
\|f\|_{b_{p, \infty ; k}^{\alpha}} \equiv \sup _{t>0} t^{-\alpha} \omega_{k}(f ; t)_{p}<\infty
$$

if $q=\infty$. Further, set

$$
B_{p, q}^{\alpha}\left(\mathbb{R}^{n}\right)=\bigcap_{k=1}^{n} B_{p, q ; k}^{\alpha}\left(\mathbb{R}^{n}\right) \quad \text { and } \quad\|f\|_{b_{p, q}^{\alpha}}=\sum_{k=1}^{n}\|f\|_{b_{p, q ; k}^{\alpha}} .
$$

We write also $B_{p, p}^{\alpha}\left(\mathbb{R}^{n}\right)=B_{p}^{\alpha}\left(\mathbb{R}^{n}\right)$.

Observe that in these definitions and notations we follow Nikol'ski1's book [23]; they can be immediately extended to anisotropic Nikol'skiı̌Besov spaces.

The spaces $B_{p}^{\alpha}\left(\mathbb{R}^{n}\right)$ are often considered as Sobolev spaces of fractional smoothness. The embedding theorem with limiting exponent for these spaces asserts that if $0<\alpha<1$ and $1 \leq p<n / \alpha$, then

$$
B_{p}^{\alpha}\left(\mathbb{R}^{n}\right) \subset L^{p_{\alpha}}\left(\mathbb{R}^{n}\right), \quad \text { where } \quad p_{\alpha}=\frac{n p}{n-\alpha p} .
$$

This theorem was proved in the late sixties independently by several authors (for the references, see [4, $\S 18]$, [14, Section 10]).

In 2002 Bourgain, Brezis and Mironescu [6] discovered that embedding $W_{p}^{1} \subset L^{p^{*}}$ can be obtained as the limit of embedding (1.2) as $\alpha \rightarrow 1$. First, they proved in [5] that for any $f \in W_{p}^{1}\left(\mathbb{R}^{n}\right)(1 \leq p<\infty)$

$$
\lim _{\alpha \rightarrow 1-}(1-\alpha)^{1 / p}\|f\|_{b_{p}^{\alpha}} \asymp\|\nabla f\|_{p}
$$

(see also [7], [18, Section 14.3], [20, Section 10.2]). The main result in [6] is the following: if $1 / 2 \leq \alpha<1$ and $1 \leq p<n / \alpha$, then for any $f \in B_{p}^{\alpha}\left(\mathbb{R}^{n}\right)$,

$$
\|f\|_{L^{p_{\alpha}}}^{p} \leq c_{n} \frac{1-\alpha}{(n-\alpha p)^{p-1}}\|f\|_{b_{p}^{\alpha}}^{p} \quad\left(p_{\alpha}=\frac{n p}{n-\alpha p}\right),
$$

where a constant $c_{n}$ depends only on $n$. In view of (1.3), inequality (1.1) is a limiting case of (1.4) as $\alpha \rightarrow 1-$. The proof of (1.4) in [6] was quite complicated. Afterwards, Maz'ya and Shaposhnikova [21] gave a simpler proof of (1.4). Moreover, they studied the limiting behaviour 
of the $B_{p}^{\alpha}$-norm and the sharp asymptotics of the embedding constant in (1.2) as $\alpha \rightarrow 0$. More precisely, they proved that

$$
\|f\|_{L^{p_{\alpha}}}^{p} \leq c_{p, n} \frac{\alpha(1-\alpha)}{(n-\alpha p)^{p-1}}\|f\|_{b_{p}^{\alpha}}^{p} \quad\left(1 \leq p<\frac{n}{\alpha}, \quad p_{\alpha}=\frac{n p}{n-\alpha p}\right) .
$$

Also, it was shown in [21] that if $f \in B_{p}^{\alpha_{0}}\left(\mathbb{R}^{n}\right)$ for some $\alpha_{0} \in(0,1)$, then

$$
\lim _{\alpha \rightarrow 0} \alpha\|f\|_{b_{p}^{\alpha}}^{p} \asymp\|f\|_{p}^{p} .
$$

We note that in the works [6] and [21] a slightly different definition of the seminorm $\|\cdot\|_{b_{p}^{\alpha}}$ was used; it is equivalent to the one given above.

Later on, it was observed in [17] that inequalities (1.4) and (1.5) can be directly derived from estimates of rearrangements obtained in [12].

Different extensions and some close aspects of these problems have been studied in [9], [10], [17], [19], [22, [25].

This paper was inspired by the results described above. Namely, it is devoted to the study of limiting behaviour of capacities in spaces $B_{p, q}^{\alpha}$ as $\alpha$ tends to 1 or $\alpha$ tends to 0 .

Let $K \subset \mathbb{R}^{n}$ be a compact set. Denote by $\mathfrak{N}(K)$ the set of all functions $f \in C_{0}^{\infty}\left(\mathbb{R}^{n}\right)$ such that $f(x) \geq 1$ for all $x \in K$. The capacity of the set $K$ in the space $W_{p}^{1}\left(\mathbb{R}^{n}\right)(1 \leq p<\infty)$ is defined by

$$
\operatorname{cap}\left(K ; W_{p}^{1}\right)=\inf \left\{\left(\sum_{k=1}^{n}\left\|D_{k} f\right\|_{p}\right)^{p}: f \in \mathfrak{N}(K)\right\}
$$

(see [20, 2.2.1]).

Similarly, let $1 \leq p, q<\infty$ and $0<\alpha<1$. The capacity of a compact set $K \subset \mathbb{R}^{n}$ in the space $B_{p, q}^{\alpha}\left(\mathbb{R}^{n}\right)$ is defined by

$$
\operatorname{cap}\left(K ; B_{p, q}^{\alpha}\right)=\inf \left\{\|f\|_{b_{p, q}^{\alpha}}^{p}: f \in \mathfrak{N}(K)\right\}
$$

(see [1], [2, Section 4], [20, Section 10.4]). Note that in this definition the $p$ th power of the Besov norm is taken. This assures that the Hausdorff dimension of the set function $\operatorname{cap}\left(\cdot ; B_{p, q}^{\alpha}\right)$ is equal to $n-\alpha p$ when $p<n / \alpha$ (see [1]).

Let $X$ denote one of the spaces $W_{p}^{1}\left(\mathbb{R}^{n}\right)$ or $B_{p, q}^{\alpha}\left(\mathbb{R}^{n}\right)$. Let $G \subset \mathbb{R}^{n}$ be an open set. Then we define the capacity of $G$ in $X$ as

$$
\operatorname{cap}(G ; X)=\sup \{\operatorname{cap}(K ; X): K \subset G, K \text { is compact }\} .
$$

The paper is organized as follows.

In Section 2 we give auxiliary statements which are used in the sequel. 
In Section 3 we prove the main result of the paper. It states that if $1 \leq p<n$ and $1 \leq q<\infty$, then for any open set $G \subset \mathbb{R}^{n}$

$$
\lim _{\alpha \rightarrow 1-}(1-\alpha)^{p / q} \operatorname{cap}\left(G ; B_{p, q}^{\alpha}\right)=\left(\frac{1}{q}\right)^{p / q} \operatorname{cap}\left(G ; W_{p}^{1}\right) .
$$

We show that this statement may fail for a compact set. If $n<p<\infty$, $n \in \mathbb{N}$, or $n=p \geq 2$, then equality (1.9) is trivially true because in these cases both the sides of (1.9) are equal to zero for any bounded open set $G$. Furthermore, (1.9) also trivially holds for $p=n=1$; in this case both the sides are equal to $2 q^{-1 / q}$ for any non-empty open bounded set $G \subset \mathbb{R}$.

In Section 4 we consider the case $\alpha \rightarrow 0$ and we prove that if $1 \leq$ $p, q<\infty$, then for any compact set $K \subset \mathbb{R}^{n}$

$$
\lim _{\alpha \rightarrow 0+} \alpha^{p / q} \operatorname{cap}\left(K ; B_{p, q}^{\alpha}\right)=2 n^{p}\left(\frac{1}{q}\right)^{p / q}|K|
$$

(as usual, $|K|$ denotes the Lebesgue measure of $K$ ). It is shown that generally this equality is not true for open sets.

\section{Auxiliary propositions}

We begin with some properties of moduli of continuity.

We shall call modulus of continuity any non-decreasing, continuous and bounded function $\omega(\delta)$ on $[0,+\infty)$ which satisfies the conditions

$$
\omega(\delta+\eta) \leq \omega(\delta)+\omega(\eta), \quad \omega(0)=0 .
$$

It is well known that for any $f \in L^{p}\left(\mathbb{R}^{n}\right)$ the functions $\omega_{j}(f ; \delta)_{p}$ are moduli of continuity.

For a modulus of continuity $\omega$ the function $\omega(\delta) / \delta$ may not be monotone. Therefore we shall use the following lemma.

Lemma 2.1. Let $\omega$ be a modulus of continuity. Set

$$
\bar{\omega}(t)=\frac{1}{t} \int_{0}^{t} \omega(u) d u, \quad t>0 .
$$

Then

$$
\bar{\omega}(t) \leq \omega(t) \leq 2 \bar{\omega}(t), \quad t>0 .
$$

Moreover, $\bar{\omega}(t)$ increases and $\bar{\omega}(t) / t$ decreases on $(0, \infty)$.

Proof. Since

$$
\bar{\omega}(t)=\int_{0}^{1} \omega(t v) d v
$$


and $\omega$ is increasing, it is obvious that $\bar{\omega}$ increases and the left-hand side inequality in (2.2) is true. We prove the right-hand side inequality in (2.2), that is,

$$
\omega(t) \leq \frac{2}{t} \int_{0}^{t} \omega(u) d u, \quad t>0
$$

We have

$$
\int_{0}^{t} \omega(u) d u=\int_{0}^{t} \omega(t-u) d u
$$

Thus, by (2.1),

$$
2 \int_{0}^{t} \omega(u) d u=\int_{0}^{t}[\omega(u)+\omega(t-u)] d u \geq t \omega(t) .
$$

This implies (2.3) . Using (2.3), we obtain

$$
\left(\frac{\bar{\omega}(t)}{t}\right)^{\prime}=-\frac{2}{t^{3}} \int_{0}^{t} \omega(u) d u+\frac{\omega(t)}{t^{2}} \leq 0 .
$$

for almost all $t>0$. Since $\bar{\omega}(t)$ is locally absolutely continuous on $(0,+\infty)$, this implies that $\bar{\omega}(t) / t$ decreases on $(0,+\infty)$.

Now we consider some estimates of partial moduli of continuity.

First, it is obvious that for any $f \in L^{p}\left(\mathbb{R}^{n}\right)(1 \leq p<\infty)$

$$
\omega_{j}(f ; \delta)_{p} \leq 2\|f\|_{p} \quad(j=1, \ldots, n) .
$$

It is easy to show that the constant 2 at the right-hand side is optimal (see Remark 4.3 below). However, for non-negative functions the constant can be improved. Namely, if $f \in L^{p}\left(\mathbb{R}^{n}\right)$ and $f(x) \geq 0$, then

$$
\omega_{j}(f ; \delta)_{p} \leq 2^{1 / p}\|f\|_{p} \quad(j=1, \ldots, n) .
$$

Indeed, let $h>0, j \in\{1, \ldots, n\}$, and set $E_{h, j}=\left\{x: f(x) \geq f\left(x+h e_{j}\right)\right\}$. Then

$$
\begin{aligned}
& \int_{\mathbb{R}^{n}}\left|f(x)-f\left(x+h e_{j}\right)\right|^{p} d x \\
& \leq \int_{E_{h, j}} f(x)^{p} d x+\int_{\mathbb{R}^{n} \backslash E_{h, j}} f\left(x+h e_{j}\right)^{p} d x \leq 2 \int_{\mathbb{R}^{n}} f(x)^{p} d x .
\end{aligned}
$$

This implies (2.5).

In what follows, for a set $E \subset \mathbb{R}^{n}$ we denote by $\chi_{E}$ its characteristic function. If $E$ is a measurable set of finite measure, then by (2.5)

$$
\omega_{j}\left(\chi_{E} ; \delta\right)_{p} \leq(2|E|)^{1 / p} .
$$

If a function $f \in L^{p}\left(\mathbb{R}^{n}\right)(1 \leq p<\infty)$ has a weak derivative $D_{j} f \in$ $L^{p}\left(\mathbb{R}^{n}\right)$ for some $1 \leq j \leq n$, then

$$
\omega_{j}(f ; \delta)_{p} \leq\left\|D_{j} f\right\|_{p} \delta
$$


(see [4, $\S 16]$ ). Moreover, by the Hardy-Littlewood theorem [23, $§ 4.8]$, if $1<p<\infty$ and $f \in L^{p}\left(\mathbb{R}^{n}\right)$, then the relation $\omega_{j}(f ; \delta)_{p}=O(\delta)$ holds if and only if there exists the weak derivative $D_{j} f \in L^{p}\left(\mathbb{R}^{n}\right)$.

We shall also use the following well-known statement which we prove for completeness.

Lemma 2.2. Let a function $f \in L^{p}\left(\mathbb{R}^{n}\right)(1 \leq p<\infty)$ have a weak derivative $D_{j} f \in L_{\text {loc }}^{1} \mathbb{R}^{n}$ for some $j \in\{1, \ldots, n\}$. Then

$$
\left\|D_{j} f\right\|_{p}=\lim _{\delta \rightarrow 0+} \frac{\omega_{j}(f ; \delta)_{p}}{\delta}=\sup _{\delta>0} \frac{\omega_{j}(f ; \delta)_{p}}{\delta} .
$$

Proof. The function $f$ can be modified on a set of measure zero so that the modified function is locally absolutely continuous on almost all straight lines parallel to the $x_{j}$-axis, and its usual derivative with respect to $x_{j}$ coincides almost everywhere on $\mathbb{R}^{n}$ with $D_{j} f$ (see [23, Chapter 4]). We assume that $f$ itself has this property. Then

$$
\frac{f\left(x+h e_{j}\right)-f(x)}{h} \rightarrow D_{j} f(x) \quad \text { as } \quad h \rightarrow 0
$$

almost everywhere on $\mathbb{R}^{n}$. Thus, by Fatou's Lemma,

$$
\begin{aligned}
& \left(\int_{\mathbb{R}^{n}}\left|D_{j} f(x)\right|^{p} d x\right)^{1 / p} \\
& \leq \varliminf_{h \rightarrow 0+}\left(h^{-p} \int_{\mathbb{R}^{n}}\left|f\left(x+h e_{j}\right)-f(x)\right|^{p} d x\right)^{1 / p} \leq \varliminf_{h \rightarrow 0+} \frac{\omega_{j}(f ; h)_{p}}{h} .
\end{aligned}
$$

On the other hand, by (2.7)

$$
\left\|D_{j} f\right\|_{p} \geq \sup _{h>0} \frac{\omega_{j}(f ; h)_{p}}{h} \geq \varlimsup_{h \rightarrow 0+} \frac{\omega_{j}(f ; h)_{p}}{h} .
$$

These inequalities yield (2.8).

Remark 2.3. As we have observed above, for a modulus of continuity $\omega$ the function $\omega(\delta) / \delta$ may not be monotone. However, it is not difficult to show that for any modulus of continuity $\omega$

$$
\lim _{\delta \rightarrow 0+} \frac{\omega(\delta)}{\delta}=\sup _{\delta>0} \frac{\omega(\delta)}{\delta} .
$$

Now we derive some estimates involving Besov norms. First, we have the following lemma which we shall often use in the sequel.

Lemma 2.4. Assume that a function $f \in L^{p}\left(\mathbb{R}^{n}\right)(1 \leq p<\infty)$ has a weak derivative $D_{j} f \in L^{p}\left(\mathbb{R}^{n}\right)$ for some $j \in\{1, \ldots, n\}$. Then $f \in$ $B_{p, q ; j}^{\alpha}\left(\mathbb{R}^{n}\right)$ for any $1 \leq q<\infty$ and any $0<\alpha<1$. Moreover,

$$
\|f\|_{b_{p, q ; j}^{\alpha}} \leq q^{-1 / q}\left[(1-\alpha)^{-1 / q} T^{1-\alpha}\left\|D_{j} f\right\|_{p}+2 \alpha^{-1 / q} T^{-\alpha}\|f\|_{p}\right]
$$


for any $T>0$.

Proof. Applying estimates (2.4) and (2.7), we obtain for $T>0$

$$
\begin{aligned}
\|f\|_{b_{p, q ; j}^{\alpha}} & \leq\left(\int_{0}^{T} t^{-\alpha q} \omega_{j}(f ; t)_{p}^{q} \frac{d t}{t}\right)^{1 / q}+\left(\int_{T}^{\infty} t^{-\alpha q} \omega_{j}(f ; t)_{p}^{q} \frac{d t}{t}\right)^{1 / q} \\
& \leq\left\|D_{j} f\right\|_{p}\left(\int_{0}^{T} t^{(1-\alpha) q} \frac{d t}{t}\right)^{1 / q}+2\|f\|_{p}\left(\int_{1}^{\infty} t^{-\alpha q} \frac{d t}{t}\right)^{1 / q} \\
& =q^{-1 / q}(1-\alpha)^{-1 / q} T^{1-\alpha}\left\|D_{j} f\right\|_{p}+2(\alpha q)^{-1 / q} T^{-\alpha}\|f\|_{p} .
\end{aligned}
$$

It is well known that for fixed $\alpha \in(0,1)$ and $p \in[1, \infty)$ the Besov spaces $B_{p, q}^{\alpha}\left(\mathbb{R}^{n}\right)$ increase as the second index $q$ increases. Moreover, the following estimate holds: if $1 \leq p<\infty, 1 \leq q<\theta \leq \infty$, and $0<\alpha<1$, then for any function $f \in L^{p}\left(\mathbb{R}^{n}\right)$ and any $j=1, \ldots, n$

$$
\|f\|_{b_{p, \theta ; j}^{\alpha}} \leq 8[\alpha(1-\alpha)]^{1 / q-1 / \theta}\|f\|_{b_{p, q ; j}^{\alpha}}
$$

(see [15, Lemma 2.2]). The constant coefficient at the right-hand side has optimal order as $\alpha \rightarrow 1$ or $\alpha \rightarrow 0$. However, the value of this coefficient can be improved. First, for "small" $\alpha$ we have the following result.

Lemma 2.5. Let $1 \leq p<\infty, 1 \leq q<\theta \leq \infty$, and $0<\alpha<1$. Then for any function $f \in L^{p}\left(\mathbb{R}^{n}\right)$ and any $j=1, \ldots, n$

$$
\|f\|_{b_{p, \theta ; j}^{\alpha}} \leq(\alpha q)^{1 / q-1 / \theta}\|f\|_{b_{p, q ; j}^{\alpha}} .
$$

Proof. Indeed, for any $\delta>0$ and any $j \in\{1, \ldots, n\}$,

$$
\begin{aligned}
& \alpha\|f\|_{b_{p, q ; j}^{\alpha}}^{q} \geq \alpha \int_{\delta}^{\infty} t^{-\alpha q} \omega_{j}(f ; t)_{p}^{q} \frac{d t}{t} \\
& \geq \omega_{j}(f ; \delta)_{p}^{q} \alpha \int_{\delta}^{\infty} t^{-\alpha q} \frac{d t}{t}=\frac{1}{q} \delta^{-\alpha q} \omega_{j}(f ; \delta)_{p}^{q} .
\end{aligned}
$$

Thus, we obtain (2.10) for $\theta=\infty$. From here, for any $\theta \in(q, \infty)$, we get

$$
\begin{aligned}
& \|f\|_{b_{p, \theta ; j}^{\alpha}}^{\theta}=\int_{0}^{\infty} t^{-\alpha \theta} \omega_{j}(f ; t)_{p}^{\theta} \frac{d t}{t} \\
& \leq\|f\|_{b_{p, \infty ; j}^{\alpha}-q}^{\theta-q} \int_{0}^{\infty} t^{-\alpha q} \omega_{j}(f ; t)_{p}^{q} \frac{d t}{t} \leq(\alpha q)^{(\theta-q) / q}\|f\|_{b_{p, q ; j}^{\alpha}}^{\theta} .
\end{aligned}
$$

This yields (2.10).

The following lemma plays an essential role in the case $\alpha \rightarrow 1-0$. 
Lemma 2.6. Let $1 \leq p<\infty, 1 \leq q<\theta \leq \infty$, and $0<\alpha<1$. Then for any function $f \in L^{p}\left(\mathbb{R}^{n}\right)$ and any $j=1, \ldots, n$

$$
\|f\|_{b_{p, \theta ; j}^{\alpha}} \leq[(1-\alpha) q]^{1 / q-1 / \theta}\left(\frac{2}{1+\alpha}\right)^{1-q / \theta}\|f\|_{b_{p, q ; j}^{\alpha}} .
$$

Proof. Fix $j \in\{1, \ldots, n\}$ and set

$$
\bar{\omega}(t)=\frac{1}{t} \int_{0}^{t} \omega_{j}(f ; u)_{p} d u, \quad t>0 .
$$

By Hardy's inequality [3, p. 124],

$$
\int_{0}^{\infty} t^{-\alpha q} \bar{\omega}(t)^{q} \frac{d t}{t} \leq \frac{1}{(1+\alpha)^{q}} \int_{0}^{\infty} t^{-\alpha q} \omega_{j}(f ; t)_{p}^{q} \frac{d t}{t} .
$$

Using this estimate, we have

$$
\begin{aligned}
\|f\|_{b_{p, q ; j}^{\alpha}}^{q} & =\int_{0}^{\infty} t^{-\alpha q} \omega_{j}(f ; t)_{p}^{q} \frac{d t}{t} \\
& \geq(1+\alpha)^{q} \int_{0}^{\infty} t^{-\alpha q} \bar{\omega}(t)^{q} \frac{d t}{t} \\
& \geq(1+\alpha)^{q} \int_{0}^{\delta} t^{(1-\alpha) q}\left(\frac{\bar{\omega}(t)}{t}\right)^{q} \frac{d t}{t}
\end{aligned}
$$

for any $\delta>0$. By Lemma 2.1, $\bar{\omega}(t) / t$ decreases on $(0,+\infty)$. Hence,

$$
\begin{aligned}
(1-\alpha)\|f\|_{b_{p, q ; j}^{\alpha}}^{q} & \geq(1+\alpha)^{q}(1-\alpha)\left(\frac{\bar{\omega}(\delta)}{\delta}\right)^{q} \int_{0}^{\delta} t^{(1-\alpha) q} \frac{d t}{t} \\
& =\frac{(1+\alpha)^{q}}{q} \delta^{-\alpha q} \bar{\omega}(\delta)^{q}, \quad \delta>0 .
\end{aligned}
$$

By (2.2),$\omega_{j}(f ; \delta)_{p} \leq 2 \bar{\omega}(\delta)$, and thus we obtain

$$
(1-\alpha)\|f\|_{b_{p, q ; j}^{\alpha}}^{q} \geq \frac{1}{q}\left(\frac{1+\alpha}{2}\right)^{q} \delta^{-\alpha q} \omega_{j}(f ; \delta)_{p}^{q}, \quad \delta>0 .
$$

This implies inequality (2.11) for $\theta=\infty$. In the case $\theta<\infty$ this inequality follows as in the proof of Lemma 2.5.

Next, we consider some estimates of distribution functions.

For any measurable function $f$ on $\mathbb{R}^{n}$, denote

$$
\lambda_{f}(y)=\left|\left\{x \in \mathbb{R}^{n}:|f(x)|>y\right\}\right|, \quad y>0 .
$$

Let $S_{0}\left(\mathbb{R}^{n}\right)$ be the class of all measurable and almost everywhere finite functions $f$ on $\mathbb{R}^{n}$ such that $\lambda_{f}(y)<\infty$ for each $y>0$. 
A non-increasing rearrangement of a function $f \in S_{0}\left(\mathbb{R}^{n}\right)$ is a nonincreasing function $f^{*}$ on $(0,+\infty)$ such that for any $y>0$

$$
\left|\left\{t>0: f^{*}(t)>y\right\}\right|=\lambda_{f}(y) .
$$

We shall assume in addition that the rearrangement $f^{*}$ is left continuous on $(0, \infty)$. Under this condition it is defined uniquely by

$$
f^{*}(t)=\inf \left\{y>0: \lambda_{f}(y)<t\right\}, \quad 0<t<\infty .
$$

It follows that

$$
f^{*}\left(\lambda_{f}(y)\right) \geq y \quad \text { for any } \quad y \geq 0 \text {. }
$$

Set also

$$
f^{* *}(t)=\frac{1}{t} \int_{0}^{t} f^{*}(u) d u
$$

For any $f \in S_{0}\left(\mathbb{R}^{n}\right)$

$$
f^{* *}(t)=\int_{t}^{\infty} \frac{f^{* *}(u)-f^{*}(u)}{u} d u, \quad t>0 .
$$

If $f \in S_{0}\left(\mathbb{R}^{n}\right)$ is locally integrable and has all weak derivatives $D_{k} f \in$ $L_{\text {loc }}^{1}(k=1, \ldots, n)$, then

$$
f^{* *}(t)-f^{*}(t) \leq n t^{1 / n} \sum_{k=1}^{n}\left(D_{k} f\right)^{* *}(t) \quad(t>0)
$$

(see [13, Lemma 5.1], [16, Lemma 3.1]).

Lemma 2.7. Let $f \in W_{p}^{1}\left(\mathbb{R}^{n}\right), 1 \leq p<n$, and let $p^{*}=n p /(n-p)$. Then

$$
\lambda_{f}(y) \leq c_{p, n}\left(\sum_{k=1}^{n}\left\|D_{k} f\right\|_{p}\right)^{p^{*}} y^{-p^{*}}, \quad y>0 .
$$

Proof. Of course, this weak-type inequality follows from the strongtype inequality (1.1). However, (2.15) is a direct consequence of the estimate (2.14). Indeed, by (2.13) and (2.14),

$$
\begin{aligned}
& f^{*}(t) \leq f^{* *}(t) \leq n \sum_{k=1}^{n} \int_{t}^{\infty} u^{1 / n-1}\left(D_{k} f\right)^{* *}(u) d u \\
& =n n^{\prime} \sum_{k=1}^{n}\left[t^{1 / n-1} \int_{0}^{t}\left(D_{k} f\right)^{*}(u) d u+\int_{t}^{\infty} u^{1 / n-1}\left(D_{k} f\right)^{*}(u) d u\right] .
\end{aligned}
$$

Applying Hölder inequality to both the integrals at the right-hand side, we have

$$
f^{*}(t) \leq c t^{-1 / p^{*}} \sum_{k=1}^{n}\left\|D_{k} f\right\|_{p}
$$


Setting $t=\lambda_{f}(y)$ and taking into account (2.12), we get (2.15).

Similarly, estimates of distribution functions in terms of moduli of continuity can be derived from the following inequality: for any $f \in$ $L^{p}\left(\mathbb{R}^{n}\right) \quad(1 \leq p<\infty)$

$$
f^{* *}(t)-f^{*}(t) \leq 2 t^{-1 / p} \sum_{k=1}^{n} \omega_{k}\left(f ; t^{1 / n}\right)_{p} .
$$

This inequality was first proved by Ul'yanov [26] in the one-dimensional case (see [14, p. 148] for an alternative proof). For all $n \geq 1$ it was proved in [11]; a simpler proof was given in [12, Theorem 1].

Lemma 2.8. Let $0<\alpha<1,1 \leq p<n / \alpha$, and $p_{\alpha}=n p /(n-\alpha p)$. Then for any function $f \in L^{p}\left(\mathbb{R}^{n}\right)$

$$
\lambda_{f}(y) \leq\left(2 p_{\alpha}\right)^{p_{\alpha}}\|f\|_{b_{p, \infty}^{\alpha}}^{p_{\alpha}} y^{-p_{\alpha}}, \quad y>0 .
$$

Proof. We have

$$
\sum_{k=1}^{n} \omega_{k}(f ; t)_{p} \leq t^{\alpha}\|f\|_{b_{p, \infty}^{\alpha}} \quad \text { for any } \quad t \geq 0 .
$$

Thus, by (2.13) and (2.16),

$$
\begin{aligned}
& f^{*}(t) \leq f^{* *}(t) \leq 2 \sum_{k=1}^{n} \int_{t}^{\infty} u^{-1 / p} \omega_{k}\left(f ; u^{1 / n}\right)_{p} \frac{d u}{u} \\
& \leq 2\|f\|_{b_{p, \infty}^{\alpha}} \int_{t}^{\infty} u^{-1 / p+\alpha / n} \frac{d u}{u}=2 p_{\alpha}\|f\|_{b_{p, \infty}^{\alpha}} t^{-1 / p_{\alpha}} .
\end{aligned}
$$

Setting $t=\lambda_{f}(y)$ and applying (2.12), we obtain (2.17).

We shall use the following notations. For any $x=\left(x_{1}, \ldots, x_{n}\right) \in \mathbb{R}^{n}$ we denote by $\widehat{x}_{k}$ the $(n-1)$-dimensional vector obtained from the $n$-tuple $x$ by removal of its $k$ th coordinate. Let $E \subset \mathbb{R}^{n}$. For every $k=1, \ldots, n$, denote by $\Pi_{k}(E)$ the orthogonal projection of $E$ onto the coordinate hyperplane $x_{k}=0$. If $E$ is a set of the type $F_{\sigma}$, then all its projections $\Pi_{k}(E)$ are sets of the type $F_{\sigma}$ in $\mathbb{R}^{n-1}$ and therefore they are measurable in $\mathbb{R}^{n-1}$. The $(n-1)$-dimensional measure of the projection $\Pi_{k}(E)$ will be denoted by $\operatorname{mes}_{n-1} \Pi_{k}(E)$. For the $n$-dimensional measure of the set $E$ we keep the usual notation $|E|$. As above, by $e_{k}$ we denote the $k$ th unit coordinate vector.

Lemma 2.9. Let $\mu, \lambda$, and $\eta$ be positive numbers and let $n \in \mathbb{N}$. Then for any set $E \subset \mathbb{R}^{n}$ of the type $F_{\sigma}$, satisfying the conditions

$$
|E| \leq \mu \quad \text { and } \quad \operatorname{mes}_{n-1} \Pi_{k}(E) \geq \lambda \quad(k=1, \ldots, n),
$$


there exists $0<h \leq 2 \mu^{2} n /(\lambda \eta)$ such that

$$
\sum_{k=1}^{n}\left|\left\{x \in E: x+h e_{k} \in E\right\}\right|<\eta .
$$

Proof. Let $E \subset \mathbb{R}^{n}$ satisfy (2.18). Denote

$$
\varphi_{E, k}(h)=\left|\left\{x \in E: x+h e_{k} \in E\right\}\right|=\int_{E} \chi_{E}\left(x+h e_{k}\right) d x \quad(h>0) .
$$

For any $H>0$ and any $k=1, \ldots, n$, we have

$$
\begin{aligned}
\int_{0}^{H} \varphi_{E, k}(h) d h & =\int_{E} d x \int_{0}^{H} \chi_{E}\left(x+h e_{k}\right) d h \\
& \leq|E| \int_{\mathbb{R}} \chi_{E}(y) d y_{k} .
\end{aligned}
$$

Integrating over projection $\Pi_{k}(E)$, we obtain

$$
\begin{aligned}
& \operatorname{mes}_{n-1} \Pi_{k}(E) \int_{0}^{H} \varphi_{E, k}(h) d h \\
& \leq|E| \int_{\Pi_{k} E} d \widehat{y}_{k} \int_{\mathbb{R}} \chi_{E}(y) d y_{k}=|E|^{2} .
\end{aligned}
$$

By (2.18), this implies that

$$
\int_{0}^{H} \varphi_{E, k}(h) \leq \frac{\mu^{2}}{\lambda} \quad(k=1, \ldots, n) .
$$

Denoting

$$
\varphi_{E}(h)=\sum_{k=1}^{n} \varphi_{E, k}(h)
$$

we have

$$
\int_{0}^{H} \varphi_{E}(h) d h \leq \frac{\mu^{2} n}{\lambda} .
$$

Thus,

$$
\inf _{h \in[0, H]} \varphi_{E}(h) \leq \frac{\mu^{2} n}{\lambda H}
$$

Setting $H=\left(2 \mu^{2} n /(\lambda \eta)\right.$, we obtain that there exists $h \in(0, H]$ (depending on $\mu, \lambda, \eta$, and $E$ ) such that $\varphi_{E}(h)<\eta$.

Throughout this paper $\mathcal{B}_{r}$ denotes the open ball with radius $r>0$ centered at the origin. In the sequel we shall use the standard mollifier (see, e.g, [18, p. 553])

$$
\varphi(x)= \begin{cases}c \exp \left(1 /\left(|x|^{2}-1\right)\right) & \text { if } \quad x \in \mathcal{B}_{1} \\ 0 & \text { if } \quad x \notin \mathcal{B}_{1},\end{cases}
$$


where $c>0$ is such that

$$
\int_{\mathbb{R}^{n}} \varphi(x) d x=1
$$

Set for $\tau>0$

$$
\varphi_{\tau}(x)=\frac{1}{\tau^{n}} \varphi\left(\frac{x}{\tau}\right) .
$$

Then $\varphi_{\tau}(x)=0$ if $|x|>\tau$, and

$$
\int_{\mathbb{R}^{n}} \varphi_{\tau}(x) d x=1
$$

We shall also use the following cutoff function

$$
\eta(x)=(\varphi * g)(x)
$$

where $g$ is the characteristic function of the open ball $\mathcal{B}_{2}$. We have that $\eta \in C_{0}^{\infty}, \eta(x)=1$ if $|x| \leq 1$ and $\eta(x)=0$ if $|x| \geq 3$.

Let $f \in C^{\infty}\left(\mathbb{R}^{n}\right) \cap W_{p}^{1}\left(\mathbb{R}^{n}\right)$. For any $\gamma>0$ the function $f_{\gamma}(x)=$ $f(x) \eta(\gamma x)$ belongs to $C_{0}^{\infty}\left(\mathbb{R}^{n}\right)$. Moreover, it is easy to see that for any $\varepsilon>0$ there exists $\gamma_{0}>0$ such that for all $0<\gamma \leq \gamma_{0}$

$$
\left\|D_{k} f_{\gamma}\right\|_{p}<\left\|D_{k} f\right\|_{p}+\varepsilon \quad(k=1, \ldots, n)
$$

(see, e.g., [24, p. 124]).

In the sequel we use also the following remark concerning capacities. Let $K \subset \mathbb{R}^{n}$ be a compact set. Denote by $\mathfrak{P}(K)$ the set of all functions $f \in C_{0}^{\infty}\left(\mathbb{R}^{n}\right)$ such that $0 \leq f(x) \leq 1$ for all $x \in \mathbb{R}^{n}$ and $f(x)=1$ in some neighborhood of $K$. It is well known that the set $\mathfrak{N}(K)$ in definitions (1.7) and (1.8) may be replaced by $\mathfrak{P}(K)$. Namely,

$$
\operatorname{cap}\left(K ; W_{p}^{1}\right)=\inf \left\{\left(\sum_{k=1}^{n}\left\|D_{k} f\right\|_{p}\right)^{p}: f \in \mathfrak{P}(K)\right\}
$$

and

$$
\operatorname{cap}\left(K ; B_{p, q}^{\alpha}\right)=\inf \left\{\|f\|_{b_{p, q}^{\alpha}}^{p}: f \in \mathfrak{P}(K)\right\}
$$

(see [20, 2.2.1]).

\section{The Limit AS $\alpha \rightarrow 1$}

In this section we prove the main result of the paper. As we have already mentioned in the Introduction, this result was inspired by the limiting relation (1.3) proved in [5]. We observe that the following slight modification of (1.3) holds: if a function $f \in L^{p}\left(\mathbb{R}^{n}\right)$ has a weak 
derivative $D_{j} f \in L^{p}\left(\mathbb{R}^{n}\right)$, then $f \in B_{p, q ; j}^{\alpha}\left(\mathbb{R}^{n}\right)$ for any $1 \leq q<\infty$ and any $0<\alpha<1$, and

$$
\lim _{\alpha \rightarrow 1-0}(1-\alpha)^{1 / q}\|f\|_{b_{p, q ; j}^{\alpha}}=\left(\frac{1}{q}\right)^{1 / q}\left\|D_{j} f\right\|_{p} .
$$

This statement follows by standard arguments from Lemma 2.2 and inequality (2.4) (see also [18, Section 14.3]).

Theorem 3.1. Let $n \geq 2,1 \leq p<n$, and $1 \leq q<\infty$. Then for any open set $G \subset \mathbb{R}^{n}$

$$
\lim _{\alpha \rightarrow 1-0}(1-\alpha)^{p / q} \operatorname{cap}\left(G ; B_{p, q}^{\alpha}\right)=\left(\frac{1}{q}\right)^{p / q} \operatorname{cap}\left(G ; W_{p}^{1}\right) .
$$

Proof. Denote

$$
\Lambda(\alpha)=(1-\alpha)^{1 / q}\left[\operatorname{cap}\left(G ; B_{p, q}^{\alpha}\right)\right]^{1 / p}, \quad 0<\alpha<1 .
$$

First we shall show that

$$
\varlimsup_{\alpha \rightarrow 1-0} \Lambda(\alpha) \leq q^{-1 / q}\left[\operatorname{cap}\left(G ; W_{p}^{1}\right)\right]^{1 / p} .
$$

We assume that $\operatorname{cap}\left(G ; W_{p}^{1}\right)<\infty$. Let $K \subset G$ be a compact set and let $0<\varepsilon<1$. There exists a function $f \in C_{0}^{\infty}\left(\mathbb{R}^{n}\right)$ such that

$$
\sum_{k=1}^{n}\left\|D_{k} f\right\|_{p}<\left(\operatorname{cap}\left(K ; W_{p}^{1}\right)+\varepsilon\right)^{1 / p}
$$

$0 \leq f(x) \leq 1$ for all $x \in \mathbb{R}^{n}$, and $f(x)=1$ in some neighborhood of $K$. Set $E_{\varepsilon}=\{x: f(x)>\varepsilon\}$. By Lemma 2.7,

$$
\left|E_{\varepsilon}\right| \leq c_{p, n}\left(\sum_{k=1}^{n}\left\|D_{k} f\right\|_{p}\right)^{p^{*}} \varepsilon^{-p^{*}}, \quad p^{*}=\frac{n p}{n-p} .
$$

Using (3.4) and taking into account that $K \subset G$, we obtain that

$$
\left|E_{\varepsilon}\right| \leq A \varepsilon^{-p^{*}}
$$

where $\left.A \equiv A(n, p, G)=c_{p, n}\left[\operatorname{cap}\left(G ; W_{p}^{1}\right)\right)+1\right]^{p^{*} / p}$. We emphasize that $A$ doesn't depend on $K$.

There exists an open set $H$ such that $K \subset H$ and $f(x)=1$ on $H$. Let $\rho$ be the distance from $K$ to the boundary of $H$ and let $0<\tau<\rho / 2$. Set

$$
f_{\varepsilon}(x)=\frac{1}{1-\varepsilon} \max (f(x)-\varepsilon, 0) \quad \text { and } \quad f_{\varepsilon, \tau}(x)=\left(f_{\varepsilon} * \varphi_{\tau}\right)(x),
$$

where $\varphi_{\tau}$ is defined by (2.21). Then $f_{\varepsilon} \in W_{p}^{1}\left(\mathbb{R}^{n}\right)$ and

$$
\left\|D_{k} f_{\varepsilon}\right\|_{p} \leq \frac{1}{1-\varepsilon}\left\|D_{k} f\right\|_{p} \quad(k=1, \ldots, n) .
$$


Furthermore, $D_{k} f_{\varepsilon, \tau}=\left(D_{k} f_{\varepsilon}\right) * \varphi_{\tau}$. Thus, by (2.22) and Young inequality,

$$
\left\|D_{k} f_{\varepsilon, \tau}\right\|_{p} \leq\left\|D_{k} f_{\varepsilon}\right\|_{p} \leq \frac{1}{1-\varepsilon}\left\|D_{k} f\right\|_{p} \quad(k=1, \ldots, n) .
$$

It is clear that $f_{\varepsilon}(x)=0$ if $x \notin E_{\varepsilon}$ and $0 \leq f_{\varepsilon}(x) \leq 1$ for all $x \in \mathbb{R}^{n}$. First, by (2.22) and (3.5), this imply that

$$
\left\|f_{\varepsilon, \tau}\right\|_{p} \leq\left\|f_{\varepsilon}\right\|_{p} \leq\left|E_{\varepsilon}\right|^{1 / p} \leq\left(A \varepsilon^{-p^{*}}\right)^{1 / p}, \quad A=A(n, p, G) .
$$

We have also that $0 \leq f_{\varepsilon, \tau}(x) \leq 1$ for all $x \in \mathbb{R}^{n}$. Furthermore, $f_{\varepsilon}(x)=$ 1 on $H$. This yields that $f_{\varepsilon, \tau}(x)=1$ for all $x$ such that $\operatorname{dist}(x, K)<\tau$. Indeed, if $\operatorname{dist}(x, K)<\tau$ and $|y| \leq \tau$, then $x-y \in H$ and $f_{\varepsilon}(x-y)=1$. Thus,

$$
f_{\varepsilon, \tau}(x)=\int_{B_{\tau}} \varphi_{\tau}(y) f_{\varepsilon}(x-y) d y=1 .
$$

Observe also that $f_{\varepsilon, \tau} \in C_{0}^{\infty}\left(\mathbb{R}^{n}\right)$. Taking into account these properties of $f_{\varepsilon, \tau}$, we have that

$$
\operatorname{cap}\left(K ; B_{p, q}^{\alpha}\right) \leq\left\|f_{\varepsilon, \tau}\right\|_{b_{p, q}^{\alpha}}^{p} .
$$

Applying Lemma 2.4 with $T=1$, we obtain

$$
(1-\alpha)^{1 / q}\left\|f_{\varepsilon, \tau}\right\|_{b_{p, q}^{\alpha}} \leq\left(\frac{1}{q}\right)^{1 / q}\left[\sum_{k=1}^{n}\left\|D_{k} f_{\varepsilon, \tau}\right\|_{p}+2\left(\frac{1-\alpha}{\alpha}\right)^{1 / q}\left\|f_{\varepsilon, \tau}\right\|_{p}\right] \text {. }
$$

Using (3.6) and (3.4) and taking into account that $K \subset G$, we have

$$
\sum_{k=1}^{n}\left\|D_{k} f_{\varepsilon, \tau}\right\|_{p} \leq \frac{1}{1-\varepsilon}\left[\operatorname{cap}\left(G ; W_{p}^{1}\right)+\varepsilon\right]^{1 / p} .
$$

The last two inequalities, together with (3.7) and (3.8), yield that

$$
\begin{aligned}
& (1-\alpha)^{1 / q}\left[\operatorname{cap}\left(K ; B_{p, q}^{\alpha}\right)\right]^{1 / p} \\
& \leq \frac{1}{q^{1 / q}(1-\varepsilon)}\left[\operatorname{cap}\left(G ; W_{p}^{1}\right)+\varepsilon\right]^{1 / p}+A^{\prime}\left(\frac{1-\alpha}{\alpha q}\right)^{1 / q} \varepsilon^{-p^{*} / p},
\end{aligned}
$$

where $A^{\prime}=2 A(n, p, G)^{1 / p}$ doesn't depend on $K$. Taking supremum over all compact sets $K \subset G$ and using notation (3.2), we get

$$
\Lambda(\alpha) \leq \frac{1}{q^{1 / q}(1-\varepsilon)}\left[\operatorname{cap}\left(G ; W_{p}^{1}\right)+\varepsilon\right]^{1 / p}+A^{\prime}\left(\frac{1-\alpha}{\alpha q}\right)^{1 / q} \varepsilon^{-p^{*} / p} .
$$

It follows that

$$
\varlimsup_{\alpha \rightarrow 1-0} \Lambda(\alpha) \leq \frac{1}{q^{1 / q}(1-\varepsilon)}\left[\operatorname{cap}\left(G ; W_{p}^{1}\right)+\varepsilon\right]^{1 / p} .
$$

Since $\varepsilon \in(0,1)$ is arbitrary, this implies (3.3) . 
Now we shall prove that

$$
\varliminf_{\alpha \rightarrow 1-0} \Lambda(\alpha) \geq q^{-1 / q}\left[\operatorname{cap}\left(G ; W_{p}^{1}\right)\right]^{1 / p} .
$$

Let $K \subset G$ be a compact set. Choose $\tau>0$ such that

$$
K_{\tau}=\left\{x \in \mathbb{R}^{n}: \operatorname{dist}(x, K) \leq 2 \tau\right\} \subset G .
$$

Then $K_{\tau}$ is compact.

We assume that $\underline{\lim }_{\alpha \rightarrow 1-0} \Lambda(\alpha)<\infty$. There exists an increasing sequence $\left\{\alpha_{\nu}\right\}$ of numbers $\alpha_{\nu} \in(0,1)$ such that $\alpha_{\nu} \rightarrow 1$ and

$$
\lim _{\nu \rightarrow \infty} \Lambda\left(\alpha_{\nu}\right)=\varliminf_{\alpha \rightarrow 1-0} \Lambda(\alpha) \text {. }
$$

We assume also that

$$
\Lambda\left(\alpha_{\nu}\right) \leq \varliminf_{\alpha \rightarrow 1-0} \Lambda(\alpha)+1 \quad(\nu \in \mathbb{N}) .
$$

For any $\nu \in \mathbb{N}$ there exists a function $f_{\nu} \in C_{0}^{\infty}\left(\mathbb{R}^{n}\right)$ such that $0 \leq$ $f_{\nu}(x) \leq 1$ for all $x \in \mathbb{R}^{n}, f_{\nu}(x)=1$ for all $x \in K_{\tau}$, and

$$
\left\|f_{\nu}\right\|_{b_{p, q}^{\alpha_{\nu}}} \leq \operatorname{cap}\left(K_{\tau} ; B_{p, q}^{\alpha}\right)^{1 / p}+\frac{1}{\nu}
$$

Since $K_{\tau} \subset G$, then $\operatorname{cap}\left(K_{\tau} ; B_{p, q}^{\alpha}\right) \leq \operatorname{cap}\left(G ; B_{p, q}^{\alpha}\right)$, and we have

$$
\left(1-\alpha_{\nu}\right)^{1 / q}\left\|f_{\nu}\right\|_{b_{p, q}^{\alpha_{\nu}}} \leq \Lambda\left(\alpha_{\nu}\right)+\frac{1}{\nu}
$$

We shall estimate $\omega_{j}\left(f_{\nu} ; \delta\right)_{p}$. Using (3.13) and Lemma 2.6 with $\theta=\infty$, we obtain that

$$
\Lambda\left(\alpha_{\nu}\right)+\frac{1}{\nu} \geq q^{-1 / q} \frac{1+\alpha_{\nu}}{2} \delta^{-\alpha_{\nu} q} \sum_{j=1}^{n} \omega_{j}\left(f_{\nu} ; \delta\right)_{p}
$$

for any $\delta>0$ and any $\nu \in \mathbb{N}$. In particular, (3.14) and (3.12) yield that

$$
\sum_{k=1}^{n} \omega_{k}\left(f_{\nu} ; \delta\right)_{p} \leq A \delta^{\alpha_{\nu}}, \quad \delta>0
$$

where $A=2 q^{1 / q}\left(\underline{\lim }_{\alpha \rightarrow 1-0} \Lambda(\alpha)+2\right)$ depends only on $p, q, n$, and $G$. To get also a control of $L^{p}$-norms, we apply truncation to the functions $f_{\nu}$. Let $0<\varepsilon<1 / 2$. Set

$$
E_{\nu, \varepsilon}=\left\{x \in \mathbb{R}^{n}: f_{\nu}(x)>\varepsilon\right\} .
$$

Let $p^{*}=n p /(n-p)$ and $p_{\nu}=n p /\left(n-\alpha_{\nu} p\right)$; then $p_{\nu}<p^{*}$. By Lemma 2.8 ,

$$
\left|E_{\nu, \varepsilon}\right| \leq\left(2 p_{\nu}\right)^{p_{\nu}} \varepsilon^{-p_{\nu}}|| f_{\nu}\left\|_{b_{p, \infty}^{\alpha_{\nu}}}^{p_{\nu}} \leq\left(2 p^{*}\right)^{p^{*}} \varepsilon^{-p^{*}}\right\| f_{\nu} \|_{b_{p, \infty}^{\alpha_{\nu}}}^{p_{\nu}}
$$


Thus, using (3.15), we obtain

$$
\left|E_{\nu, \varepsilon}\right| \leq A^{\prime} \varepsilon^{-p^{*}} \quad(\nu \in \mathbb{N}),
$$

where $A^{\prime}$ depends only on $p, q, n$, and $G, A^{\prime}=\left(2 p^{*} A\right)^{p^{*}}$. Set now

$$
f_{\nu, \varepsilon}(x)=\frac{1}{1-\varepsilon} \max \left(f_{\nu}(x)-\varepsilon, 0\right) .
$$

It is easily seen that

$$
\omega_{j}\left(f_{\nu, \varepsilon} ; \delta\right)_{p} \leq \frac{1}{1-\varepsilon} \omega_{j}\left(f_{\nu} ; \delta\right)_{p}, \quad \delta \geq 0 \quad(j=1, \ldots, n) .
$$

Moreover, $0 \leq f_{\nu, \varepsilon}(x) \leq 1$ for all $x \in \mathbb{R}^{n}, f_{\nu, \varepsilon}(x)=1$ for all $x \in K_{\tau}$, and $f_{\nu, \varepsilon}(x)=0$ for $x \notin E_{\nu, \varepsilon}$. Applying (3.16), we get

$$
\left\|f_{\nu, \varepsilon}\right\|_{p}^{p} \leq\left|E_{\nu, \varepsilon}\right| \leq A^{\prime} \varepsilon^{-p^{*}} \quad(\nu \in \mathbb{N})
$$

Besides, by (3.15) and (3.17),

$$
\omega\left(f_{\nu, \varepsilon} ; \delta\right)_{p} \leq 2 A^{\prime} \delta^{\alpha_{1}}, \quad \delta \in[0,1], \nu \in \mathbb{N} .
$$

By virtue of (3.18), (3.19), and the compactness criterion (see [8, p. $111]$ ), for any compact set $Q \subset \mathbb{R}^{n}$ there exists a subsequence of $\left\{f_{\nu, \varepsilon}\right\}$ that converges in $L^{p}(Q)$. Therefore, by Riesz's theorem, for any compact set $Q \subset \mathbb{R}^{n}$ there exists a subsequence of $\left\{f_{\nu, \varepsilon}\right\}$ that converges almost everywhere on $Q$. Let $Q_{s}=[-s, s]^{n}, s \in \mathbb{N}$. A successive extraction of subsequences gives strictly increasing sequences $\left\{\nu_{m}^{(s)}\right\}$ $(s=1,2, \ldots)$ of natural numbers such that

$$
\left\{\nu_{m}^{(1)}\right\} \supset\left\{\nu_{m}^{(2)}\right\} \supset \ldots \supset\left\{\nu_{m}^{(s)}\right\} \supset \ldots
$$

and for each $s \in N$ the subsequence $\left\{f_{\nu_{m}^{(s)}, \varepsilon}\right\}$ converges almost everywhere on $Q_{s}$. Then the diagonal subsequence $\left\{f_{\nu_{s}^{(s)}, \varepsilon}\right\}$ converges almost everywhere on $\mathbb{R}^{n}$. For simplicity, we assume that $\left\{f_{\nu, \varepsilon}\right\}$ itself converges almost everywhere on $\mathbb{R}^{n}$. Let

$$
f_{\varepsilon}(x)=\lim _{\nu \rightarrow \infty} f_{\nu, \varepsilon}(x) .
$$

Since $f_{\nu, \varepsilon}(x)=1$ on $K_{\tau}$ for any $\nu \in \mathbb{N}$, then

$$
f_{\varepsilon}(x)=1 \text { for all } x \in K_{\tau} \text {. }
$$

We have also that $0 \leq f_{\varepsilon}(x) \leq 1$ almost everywhere on $\mathbb{R}^{n}$. Further, by Fatou's lemma and (3.18)

$$
\left\|f_{\varepsilon}\right\|_{p}^{p} \leq A^{\prime} \varepsilon^{-p^{*}}
$$

Fatou's lemma yields also that for any $h>0$ and any $j=1, \ldots, n$

$$
\int_{\mathbb{R}^{n}}\left|f_{\varepsilon}\left(x+h e_{j}\right)-f_{\varepsilon}(x)\right|^{p} d x \leq \varliminf_{\nu \rightarrow \infty} \int_{\mathbb{R}^{n}}\left|f_{\nu, \varepsilon}\left(x+h e_{j}\right)-f_{\nu, \varepsilon}(x)\right|^{p} d x .
$$


Thus,

$$
\omega_{j}\left(f_{\varepsilon} ; \delta\right)_{p} \leq \varliminf_{\nu \rightarrow \infty} \omega_{j}\left(f_{\nu, \varepsilon} ; \delta\right)_{p}, \quad \delta \geq 0(j=1, \ldots, n) .
$$

Let $\varphi_{\tau}$ be the mollifier defined by (2.21). Set $f_{\varepsilon, \tau}=f_{\varepsilon} * \varphi_{\tau}$. Clearly, $0 \leq f_{\varepsilon, \tau}(x) \leq 1$ for all $x \in \mathbb{R}^{n}$ and, by (2.22) and (3.20),

$$
f_{\varepsilon, \tau}(x)=1 \quad \text { if } \quad \operatorname{dist}(x, K)<\tau .
$$

Besides, by Young inequality and (2.22),

$$
\omega_{j}\left(f_{\varepsilon, \tau} ; \delta\right)_{p} \leq \omega_{j}\left(f_{\varepsilon} ; \delta\right)_{p}, \quad \delta \geq 0(j=1, \ldots, n) .
$$

Applying inequalities (3.14) and (3.17), we obtain

$$
\Lambda\left(\alpha_{\nu}\right)+\frac{1}{\nu} \geq \frac{\left(1+\alpha_{\nu}\right)(1-\varepsilon)}{2 q^{1 / q}} \delta^{-\alpha_{\nu}} \sum_{j=1}^{n} \omega_{j}\left(f_{\nu, \varepsilon} ; \delta\right)_{p} .
$$

By (3.11), (3.22), and (3.24), this implies that

$$
\varliminf_{\alpha \rightarrow 1-0} \Lambda(\alpha) \geq \frac{1-\varepsilon}{q^{1 / q}} \sum_{j=1}^{n} \frac{\omega_{j}\left(f_{\varepsilon, \tau} ; \delta\right)_{p}}{\delta}
$$

for any $\delta>0$. Taking into account (3.21), we have $f_{\varepsilon, \tau} \in L^{p}\left(\mathbb{R}^{n}\right) \cap$ $C^{\infty}\left(\mathbb{R}^{n}\right)$. Making $\delta$ tend to zero and applying Lemma 2.2, we obtain

$$
\varliminf_{\alpha \rightarrow 1-0} \Lambda(\alpha) \geq \frac{1-\varepsilon}{q^{1 / q}} \sum_{j=1}^{n}\left\|D_{j} f_{\varepsilon, \tau}\right\|_{p} .
$$

Let $\eta$ be the cutoff function defined by (2.23)). Set $g(x)=f_{\varepsilon, \tau}(x) \eta(\gamma x)$, $\gamma>0$. Then $g \in C_{0}^{\infty}\left(\mathbb{R}^{n}\right)$ and $0 \leq g(x) \leq 1$ for all $x \in \mathbb{R}^{n}$. If $\gamma$ is sufficiently small, then, by virtue of (3.23), $g(x)=1$ if $\operatorname{dist}(x, K)<\tau$. Moreover, $\gamma$ can be chosen so small that (see (2.24))

$$
\left\|D_{j} g\right\|_{p}<\left\|D_{j} f_{\varepsilon, \tau}\right\|_{p}+\frac{\varepsilon}{n} \quad(j=1, \ldots, n) .
$$

Since

$$
\sum_{j=1}^{n}\left\|D_{j} g\right\|_{p} \geq \operatorname{cap}\left(K ; W_{p}^{1}\right)^{1 / p},
$$

inequality (3.26) yields that

$$
\varliminf_{\alpha \rightarrow 1-0} \Lambda(\alpha) \geq \frac{1-\varepsilon}{q^{1 / q}}\left[\operatorname{cap}\left(K ; W_{p}^{1}\right)^{1 / p}-\varepsilon\right] .
$$

Taking into account that $\varepsilon \in(0,1)$ and a compact set $K \subset G$ are arbitrary, we obtain inequality (3.9). Together with (3.3), this gives (3.1). 
Remark 3.2. The statement of Theorem 3.1 fails to hold for compact sets. To show it, we use a theorem on capacity of a Cantor set [2, Section 5.3]. Let $1<p<n, p=q$, and let $0<\alpha<1$. It is known that in this case the $B_{p}^{\alpha}$-capacity is equivalent to the Bessel capacity $C_{\alpha, p}[2$, p. 107]. Set

$$
l_{k}=\left((k+4)^{2} 2^{-k n}\right)^{1 /(n-p)} \quad(k=0,1, \ldots) .
$$

Then $l_{k+1}<l_{k} / 2$ for all $k \geq 0$. Further,

$$
\sum_{k=0}^{\infty} 2^{-k n} l_{k}^{p-n}<\infty \text { and } \sum_{k=0}^{\infty} 2^{-k n} l_{k}^{\alpha p-n}=\infty
$$

for any $0<\alpha<1$. Let $E$ be the Cantor set corresponding to the sequence $\left\{l_{k}\right\}$ defined in $[2,(5.3 .1)]$. It follows by [2, Theorem 5.3.2] that

$$
\operatorname{cap}\left(E ; W_{p}^{1}\right)>0 \quad \text { and } \operatorname{cap}\left(E ; B_{p}^{\alpha}\right)=0
$$

for any $0<\alpha<1$. Thus, equality (3.1) does not hold.

Remark 3.3. We observe that if $n<p<\infty, n \in \mathbb{N}$, or $p=n \geq 2$, then equality (3.1) is trivially true. It is closely related to the fact that in these cases the Sobolev capacity of a ball in $\mathbb{R}^{n}$ is equal to zero (see [20, p. 148]). For completeness, we give the corresponding arguments in detail.

First, let $n<p<\infty$. We consider the ball $\mathcal{B}_{r}, r>0$. Let $\eta$ be the cutoff function defined by (2.23). Set $f_{\gamma}(x)=\eta(\gamma x)$, where $0<\gamma<$ $1 / r$. Then $f_{\gamma} \in C_{0}^{\infty}\left(\mathbb{R}^{n}\right), 0 \leq f_{\gamma}(x) \leq 1$ for all $x \in \mathbb{R}^{n}$, and $f_{\gamma}(x)=1$ in some neighborhood of $\overline{\mathcal{B}}_{r}$. Further,

$$
\left\|D_{k} f_{\gamma}\right\|_{p}=\gamma^{1-n / p}\left\|D_{k} \eta\right\|_{p} \quad(k=1, \ldots, n) .
$$

This implies that $\operatorname{cap}\left(\mathcal{B}_{r} ; W_{p}^{1}\right)=0$. Moreover, if $n / p<\alpha<1$, then we have also that

$$
\operatorname{cap}\left(\mathcal{B}_{r} ; B_{p, q}^{\alpha}\right)=0
$$

for any $1 \leq q<\infty$. Indeed,

$$
\operatorname{cap}\left(\mathcal{B}_{r} ; B_{p, q}^{\alpha}\right) \leq\left\|f_{\gamma}\right\|_{b_{p, q}^{\alpha}}^{p} .
$$

Thus, applying Lemma 2.4 and (3.27), we obtain

$$
\begin{aligned}
& \left\|f_{\gamma}\right\|_{b_{, q ;}^{\alpha} ; k} \leq q^{-1 / q}\left[(1-\alpha)^{-1 / q} T^{1-\alpha}\left\|D_{k} f_{\gamma}\right\|_{p}+2 \alpha^{-1 / q} T^{-\alpha}\left\|f_{\gamma}\right\|_{p}\right] \\
& \leq((1-\alpha) q)^{-1 / q}\left\|D_{k} \eta\right\|_{p} T^{1-\alpha} \gamma^{1-n / p}+2(\alpha q)^{-1 / q}\|\eta\|_{p} T^{-\alpha} \gamma^{-n / p}
\end{aligned}
$$

for any $T>0$ and any $1 \leq k \leq n$. Setting $T=1 / \gamma$, we get

$$
\left\|f_{\gamma}\right\|_{b_{p, q ; k}^{\alpha}} \leq\left[((1-\alpha) q)^{-1 / q}\left\|D_{k} \eta\right\|_{p}+2(\alpha q)^{-1 / q}\|\eta\|_{p}\right] \gamma^{\alpha-n / p} .
$$


Since $0<\gamma<1 / r$ is arbitrary and $\alpha>n / p$, this implies (3.28). Thus, if $p>n$, then for any open set $G \subset \mathbb{R}^{n}$ both the capacities in relation (3.1) are equal to 0.

Let now $p=n \geq 2$. We have $\operatorname{cap}\left(\mathcal{B}_{r} ; W_{n}^{1}\right)=0(r>0)[20$, p. 148]). At the same time, it follows from Lemma 2.8 and inequality (2.9) that $\operatorname{cap}\left(\mathcal{B}_{r} ; B_{n, q}^{\alpha}\right)>0$ for any $0<\alpha<1$ and any $1 \leq q<\infty$. Nevertheless, we shall show that

$$
\lim _{\alpha \rightarrow 0}(1-\alpha)^{n / q} \operatorname{cap}\left(\mathcal{B}_{r} ; B_{n, q}^{\alpha}\right)=0 \quad(r>0) .
$$

Let $\sigma=(n-1) /(2 n)$ and set

$$
f_{0}(x)=\left\{\begin{array}{lll}
|\ln | x||^{\sigma} & \text { if } & |x| \leq 1 \\
0 & \text { if } & |x|>1
\end{array}\right.
$$

It is easy to see that $f \in W_{n}^{1}\left(\mathbb{R}^{n}\right)$. Let $\varepsilon>0$. Set $f_{1}(x)=\min \left(\varepsilon f_{0}(x), 1\right)$. Since $f_{0}(x) \rightarrow+\infty$ as $x \rightarrow 0$, there exists a closed ball $U_{\varepsilon}$ centered at the origin such that $f_{1}(x)=1$ for all $x \in U_{\varepsilon}$. There is $\gamma>0$ such that $\gamma x \in U_{\varepsilon}$ for all $x \in \overline{\mathcal{B}}_{r+1}$. Set $f_{2}(x)=f_{1}(\gamma x)$. Then

$$
\left\|D_{k} f_{2}\right\|_{n}=\left\|D_{k} f_{1}\right\|_{n} \leq \varepsilon\left\|D_{k} f_{0}\right\|_{n} \quad(k=1, \ldots, n)
$$

and

$$
\left\|f_{2}\right\|_{n}=\frac{\left\|f_{1}\right\|_{n}}{\gamma} \leq \frac{\varepsilon\left\|f_{0}\right\|_{n}}{\gamma}
$$

Finally, we define $f=f_{2} * \varphi_{1 / 2}$ (see (2.21) ). Then $f \in C_{0}^{\infty}\left(\mathbb{R}^{n}\right)$, $f(x)=1$ in $\mathcal{B}_{r+1 / 2}$, and $0 \leq f(x) \leq 1$ for all $x \in \mathbb{R}^{n}$. Moreover,

$$
\left\|D_{k} f\right\|_{n} \leq \varepsilon\left\|D_{k} f_{0}\right\|_{n} \quad(k=1, \ldots, n)
$$

and

$$
\|f\|_{n} \leq \frac{\varepsilon\left\|f_{0}\right\|_{n}}{\gamma}
$$

First, this shows that $\operatorname{cap}\left(\mathcal{B}_{r} ; W_{n}^{1}\right)=0$. Further, applying Lemma 2.4 with $T=1$ and using (3.30) and (3.31), we obtain

$$
\begin{aligned}
(1-\alpha)^{1 / q}\|f\|_{b_{n, q ; k}^{\alpha}} & \leq q^{-1 / q}\left(\left\|D_{k} f\right\|_{n}+2\left(\frac{1-\alpha}{\alpha}\right)^{1 / q}\|f\|_{n}\right) \\
& \leq \varepsilon q^{-1 / q}\left(\left\|D_{k} f_{0}\right\|_{n}+\frac{2}{\gamma}\left(\frac{1-\alpha}{\alpha}\right)^{1 / q}\left\|f_{0}\right\|_{n}\right)
\end{aligned}
$$

Since cap $\left(\mathcal{B}_{r} ; B_{n, q}^{\alpha}\right) \leq\left\|f_{\gamma}\right\|_{b_{n, q}^{\alpha}}^{n}$, this implies that

$$
\varlimsup_{\alpha \rightarrow 0}(1-\alpha)^{1 / q} \operatorname{cap}\left(\mathcal{B}_{r} ; B_{n, q}^{\alpha}\right)^{1 / n} \leq \varepsilon q^{-1 / q} \sum_{k=1}^{n}\left\|D_{k} f_{0}\right\|_{n} .
$$


By view of the arbitrariness of $\varepsilon>0$, we obtain (3.29). Thus, for $p=n \geq 2$ (3.1) also is trivially true.

Remark 3.4. The remaining case $p=n=1$ is also "degenerate". First, if a set $E$ consists of one point, $E=\left\{x_{0}\right\}$, then $\operatorname{cap}\left(E ; W_{1}^{1}\right) \geq 2$. Indeed, if $f \in C_{0}^{\infty}(\mathbb{R})$ and $f\left(x_{0}\right)=1$, then

$$
-\int_{x_{0}}^{\infty} f^{\prime}(x) d x=\int_{-\infty}^{x_{0}} f^{\prime}(x) d x=1 .
$$

Thus, $\left\|f^{\prime}\right\|_{1} \geq 2$. Further, let $K \subset \mathbb{R}$ be an arbitrary compact set, $K \subset[-a, a](a>0)$. Set

$$
f_{a}(x)=\left\{\begin{array}{lll}
1 & \text { if } & |x| \leq a \\
(a / x)^{2} & \text { if } & |x|>a .
\end{array}\right.
$$

Then $f_{a} \in W_{1}^{1}(\mathbb{R})$ and $\left\|f_{a}^{\prime}\right\|_{1}=2$. We obtain that $\operatorname{cap}\left(K ; W_{1}^{1}\right)=2$ for any compact set $K \neq \emptyset$, and therefore $\operatorname{cap}\left(G ; W_{1}^{1}\right)=2$ for any non-empty open set $G \subset \mathbb{R}$.

Now we observe that for any $f \in L^{1}(\mathbb{R})$ and any $h>0$

$$
\begin{aligned}
& \int_{0}^{\infty}|f(x)-f(x+h)| d x \\
& \geq \int_{0}^{\infty}|f(x)| d x-\int_{0}^{\infty}|f(x+h)| d x=\int_{0}^{h}|f(x)| d x
\end{aligned}
$$

and similarly

$$
\int_{-\infty}^{0}|f(x)-f(x+h)| d x \geq \int_{0}^{h}|f(x)| d x .
$$

Thus,

$$
\omega(f ; h)_{1} \geq 2 \int_{0}^{h}|f(x)| d x \quad(h>0) .
$$

Let $I=\left[-h_{0}, h_{0}\right]\left(h_{0}>0\right)$. Let $f \in L^{1}(\mathbb{R})$ and $f(x)=1$ on $I$. Then, by (3.33),$\omega(f ; h)_{1} \geq 2 h$ for all $0 \leq h \leq h_{0}$. Thus, for any $1 \leq q<\infty$

$$
\begin{aligned}
(1-\alpha)\|f\|_{b_{1, q}^{\alpha}}^{q} & \geq(1-\alpha) \int_{0}^{h_{0}} h^{-\alpha q} \omega(f ; h)_{1}^{q} \frac{d h}{h} \\
& \geq 2^{q}(1-\alpha) \int_{0}^{h_{0}} h^{(1-\alpha) q} \frac{d h}{h}=\frac{2^{q}}{q} h_{0}^{(1-\alpha) q} .
\end{aligned}
$$

This implies that

$$
\varliminf_{\alpha \rightarrow 1-}(1-\alpha)^{1 / q} \operatorname{cap}\left(G ; B_{1, q}^{\alpha}\right) \geq 2 q^{-1 / q}
$$


for any open set $G \subset \mathbb{R}$. On the other hand, assume that $G \subset$ $[-a, a](a>0)$. Applying Lemma 2.4 to the function (3.32), we have

$$
\begin{aligned}
(1-\alpha)^{1 / q}\left\|f_{a}\right\|_{b_{1, q}^{\alpha}} & \leq q^{-1 / q}\left\|f_{a}^{\prime}\right\|_{1}+2\left(\frac{1-\alpha}{\alpha q}\right)^{1 / q}\left\|f_{a}\right\|_{1} \\
& =q^{-1 / q}\left[2+8 a\left(\frac{1-\alpha}{\alpha}\right)^{1 / q}\right] .
\end{aligned}
$$

It follows that

$$
\varlimsup_{\alpha \rightarrow 1-}(1-\alpha)^{1 / q} \operatorname{cap}\left(G ; B_{1, q}^{\alpha}\right) \leq 2 q^{-1 / q} .
$$

Thus, for any open bounded set $G \subset \mathbb{R}$

$$
\lim _{\alpha \rightarrow 1-}(1-\alpha)^{1 / q} \operatorname{cap}\left(G ; B_{1, q}^{\alpha}\right)=q^{-1 / q} \operatorname{cap}\left(G ; W_{1}^{1}\right)=2 q^{-1 / q} .
$$

\section{The LIMIT AS $\alpha \rightarrow 0$}

In this section we study the behaviour of $B_{p, q}^{\alpha}$-capacities as $\alpha \rightarrow 0$ (cf. (1.6) and Remark 4.3 below).

Theorem 4.1. Let $1 \leq p<\infty$ and $1 \leq q<\infty$. Then for any compact set $K \subset \mathbb{R}^{n}$

$$
\lim _{\alpha \rightarrow 0+} \alpha^{p / q} \operatorname{cap}\left(K ; B_{p, q}^{\alpha}\right)=2 n^{p}\left(\frac{1}{q}\right)^{p / q}|K| .
$$

Proof. Denote

$$
\Lambda(\alpha)=\alpha^{1 / q}\left[\operatorname{cap}\left(K ; B_{p, q}^{\alpha}\right)\right]^{1 / p}, \quad 0<\alpha<1 .
$$

First we prove that

$$
\varliminf_{\alpha \rightarrow 0+} \Lambda(\alpha) \geq n 2^{1 / p} q^{-1 / q}|K|^{1 / p}
$$

We assume that $|K|>0$. It is clear that $\underline{\lim }_{\alpha \rightarrow 0+} \Lambda(\alpha)<\infty$. There exists a decreasing sequence $\left\{\alpha_{\nu}\right\}$ of numbers $\alpha_{\nu} \in(0,1 / 2]$ with $\alpha_{1}=$ $\min (1, n / p) / 2$ such that $\alpha_{\nu} \rightarrow 0$ and

$$
\lim _{\nu \rightarrow \infty} \Lambda\left(\alpha_{\nu}\right)=\underset{\alpha \rightarrow 0+}{\lim _{\alpha \rightarrow 0}} \Lambda(\alpha) .
$$

We emphasize that $\alpha_{\nu}<n / p$ for all $\nu \in \mathbb{N}$. We may assume that

$$
\Lambda\left(\alpha_{\nu}\right) \leq \lim _{\alpha \rightarrow 0+} \Lambda(\alpha)+1 \quad(\nu \in \mathbb{N})
$$

For any $\nu \in \mathbb{N}$ there exists a function $f_{\nu} \in C_{0}^{\infty}\left(\mathbb{R}^{n}\right)$ such that $0 \leq$ $f_{\nu}(x) \leq 1$ for all $x \in \mathbb{R}^{n}, f_{\nu}(x)=1$ for all $x \in K$, and

$$
\Lambda\left(\alpha_{\nu}\right) \geq \alpha_{\nu}^{1 / q}|| f_{\nu} \|_{b_{p, q}^{\alpha_{\nu}}}-\frac{1}{\nu}
$$


Applying Lemma 2.5 for $\theta=\infty$, we obtain that

$$
\Lambda\left(\alpha_{\nu}\right)+\frac{1}{\nu} \geq q^{-1 / q} t^{-\alpha_{\nu}} \sum_{j=1}^{n} \omega_{j}\left(f_{\nu} ; t\right)_{p}
$$

for any $t>0$ and any $\nu \in \mathbb{N}$. In particular, by (4.4) and (4.5),

$$
\sum_{j=1}^{n} \omega_{j}\left(f_{\nu} ; t\right)_{p} \leq A t^{\alpha_{\nu}}, \quad t>0
$$

where $A$ depends only on $p, q, n$, and $K$.

Let $0<\varepsilon<1$. Set

$$
E_{\nu, \varepsilon}=\left\{x \in \mathbb{R}^{n}: f_{\nu}(x)>\varepsilon\right\} .
$$

Denote $p_{\nu}=n p /\left(n-\alpha_{\nu} p\right)$. Then $p_{\nu} \leq p_{1}$. By Lemma 2.8,

$$
\left|E_{\nu, \varepsilon}\right| \leq\left.\left(2 p_{\nu}\right)^{p_{\nu}} \varepsilon^{-p_{\nu}}|| f_{\nu}\right|_{b_{p, \infty}^{\alpha_{\nu}}} ^{p_{\nu}} \leq\left.\left(2 p_{1}\right)^{p_{1}} \varepsilon^{-p_{1}}|| f_{\nu}\right|_{b_{p, \infty}^{\alpha_{\nu}}} ^{p_{\nu}}
$$

Thus, using (4.6), we obtain that

$$
\left|E_{\nu, \varepsilon}\right| \leq A^{\prime} \varepsilon^{-p_{1}} \quad(\nu \in \mathbb{N}),
$$

where $A^{\prime}$ depends only on $p, q, n$, and $K$.

Since $|K|>0$, there exists a number $\lambda(K)>0$ such that

$$
\operatorname{mes}_{n-1} \Pi_{j}(K) \geq \lambda(K) \text { for all } 1 \leq j \leq n .
$$

Further, $K \subset E_{\nu, \varepsilon}$, and thus

$$
\operatorname{mes}_{n-1} \Pi_{j}\left(E_{\nu, \varepsilon}\right) \geq \lambda(K) \quad(\nu \in \mathbb{N}, j=1, \ldots, n) .
$$

Now we apply Lemma 2.9 with $\mu=A^{\prime} \varepsilon^{-p_{1}}, \lambda=\lambda(K)$, and $\eta=\varepsilon|K|$. Set $H=2 \mu^{2} n /(\lambda \eta)$. By Lemma 2.9, for any $\nu \in \mathbb{N}$ there exists $h_{\nu} \in$ $(0, H]$ such that

$$
\sum_{j=1}^{n}\left|\left\{x \in E_{\nu, \varepsilon}: x+h_{\nu} e_{j} \in E_{\nu, \varepsilon}\right\}\right|<\varepsilon|K| .
$$

We emphasize that $H$ doesn't depend on $\nu$. Denote

$$
K_{j}^{(\nu)}=\left\{x \in \mathbb{R}^{n}: x+h_{\nu} e_{j} \in K\right\} .
$$

Since $K \subset E_{\nu, \varepsilon}(\nu \in \mathbb{N})$, we derive from (4.8) that for any $\nu \in \mathbb{N}$ and any $j=1, \ldots, n$

$$
\begin{gathered}
\left|\left\{x \in K: f_{\nu}\left(x+h_{\nu} e_{j}\right) \leq \varepsilon\right\}\right|>(1-\varepsilon)|K|, \\
\left|\left\{x \in K_{j}^{(\nu)}: f_{\nu}(x) \leq \varepsilon\right\}\right|>(1-\varepsilon)|K|,
\end{gathered}
$$


and $\left|K \cap K_{j}^{(\nu)}\right|<\varepsilon|K|$. Thus, taking into account that $0 \leq f_{\nu}(x) \leq 1$ and $f_{\nu}(x)=1$ on $K$, we obtain

$$
\begin{aligned}
& \omega_{j}\left(f_{\nu} ; H\right)_{p}^{p} \geq \int_{K}\left|f_{\nu}(x)-f_{\nu}\left(x+h_{\nu} e_{j}\right)\right|^{p} d x \\
& +\int_{K_{j}^{(\nu)}}\left|f_{\nu}(x)-f_{\nu}\left(x+h_{\nu} e_{j}\right)\right|^{p} d x-\varepsilon|K| \geq 2(1-\varepsilon)^{p+1}|K|-\varepsilon|K|
\end{aligned}
$$

for all $1 \leq j \leq n$. From here and (4.5),

$$
\begin{aligned}
\Lambda\left(\alpha_{\nu}\right)+\frac{1}{\nu} & \geq q^{-1 / q} H^{-\alpha_{\nu}} \sum_{j=1}^{n} \omega_{j}\left(f_{\nu} ; H\right)_{p} \\
& \geq n q^{-1 / q} H^{-\alpha_{\nu}}\left[\left(2(1-\varepsilon)^{p+1}-\varepsilon\right)|K|\right]^{1 / p}
\end{aligned}
$$

By (4.3), this implies that

$$
\varliminf_{\alpha \rightarrow 0+} \Lambda(\alpha) \geq n q^{-1 / q}\left[\left(2(1-\varepsilon)^{p+1}-\varepsilon\right)|K|\right]^{1 / p} .
$$

Since $\varepsilon \in(0,1)$ is arbitrary, this yields (4.2).

Now we shall prove that

$$
\varlimsup_{\alpha \rightarrow 0+} \Lambda(\alpha) \leq n 2^{1 / p} q^{-1 / q}|K|^{1 / p}
$$

Set for $\tau>0$

$$
K_{\tau}=\left\{x \in \mathbb{R}^{n}: \operatorname{dist}(x, K) \leq 2 \tau\right\} .
$$

Fix $0<\varepsilon<1$ and choose $\tau>0$ such that

$$
\left|K_{\tau}\right|<|K|+\varepsilon \text {. }
$$

Let $\varphi_{\tau}$ be the standard mollifier defined by (2.20). Set

$$
f_{\tau}=\chi_{\tau} * \varphi_{\tau}
$$

where $\chi_{\tau}$ is the characteristic function of the set $K_{\tau}$. Then $f_{\tau} \in C_{0}^{\infty}\left(\mathbb{R}^{n}\right)$, $0 \leq f_{\tau}(x) \leq 1$ for all $x \in \mathbb{R}^{n}$, and $f_{\tau}(x)=1$ for all $x$ such that $\operatorname{dist}(x, K) \leq \tau$. Thus,

$$
\operatorname{cap}\left(K ; B_{p, q}^{\alpha}\right) \leq\left\|f_{\tau}\right\|_{b_{p, q}^{\alpha}}^{p} .
$$

Using (2.6) and (2.22), we have

$$
\omega_{j}\left(f_{\tau} ; t\right)_{p} \leq \omega_{j}\left(\chi_{\tau} ; t\right)_{p} \leq\left(2\left|K_{\tau}\right|\right)^{1 / p} \leq[2(|K|+\varepsilon)]^{1 / p} .
$$


Applying (2.7) and (4.13), we obtain

$$
\begin{aligned}
& \alpha^{1 / q}\left\|f_{\tau}\right\|_{b_{p, q}^{\alpha}}=\alpha^{1 / q} \sum_{j=1}^{n}\left(\int_{0}^{\infty} t^{-\alpha q} \omega_{j}\left(f_{\tau} ; t\right)_{p}^{q} \frac{d t}{t}\right)^{1 / q} \\
& \leq \alpha^{1 / q} \sum_{j=1}^{n}\left[\left\|D_{j} f_{\tau}\right\|_{p}\left(\int_{0}^{1} t^{(1-\alpha) q} \frac{d t}{t}\right)^{1 / q}+\left(\int_{1}^{\infty} t^{-\alpha q} \omega_{j}\left(f_{\tau} ; t\right)_{p}^{q} \frac{d t}{t}\right)^{1 / q}\right] \\
& \leq\left(\frac{\alpha}{(1-\alpha) q}\right)^{1 / q} \sum_{j=1}^{n}\left\|D_{j} f_{\tau}\right\|_{p}+n 2^{1 / p} q^{-1 / q}(|K|+\varepsilon)^{1 / p} .
\end{aligned}
$$

This estimate and (4.12) imply that

$$
\Lambda(\alpha) \leq\left(\frac{\alpha}{(1-\alpha) q}\right)^{1 / q} \sum_{j=1}^{n}\left\|D_{j} f_{\tau}\right\|_{p}+2^{1 / p} q^{-1 / q} n(|K|+\varepsilon)^{1 / p} .
$$

It follows that

$$
\varlimsup_{\alpha \rightarrow 0+} \Lambda(\alpha) \leq n 2^{1 / p} q^{-1 / q}(|K|+\varepsilon)^{1 / p} .
$$

Since $\varepsilon \in(0,1)$ is arbitrary, this implies (4.9). Inequalities (4.2) and (4.9) yield (4.1).

Remark 4.2. Generally, Theorem 4.1 fails to hold for open sets. As in Section 3, we shall show it using Cantor sets [2, Section 5.3].

Let $f \in B_{p}^{\alpha}\left(\mathbb{R}^{n}\right)$ an let $\delta_{\lambda} f(x)=f(\lambda x)(\lambda>0)$ be a dilation of $f$. It is easily seen that

$$
\left\|\delta_{\lambda} f\right\|_{b_{p}^{\alpha}}^{p}=\lambda^{\alpha p-n}\|f\|_{b_{p}^{\alpha}}^{p}
$$

Assume that $p>1$ and $0<\alpha<\min (1, n / p)$. Recall that in this case the $B_{p}^{\alpha}$-capacity is equivalent to the Bessel capacity $C_{\alpha, p}$ [2, p. 107]. There exists $k_{0}=k_{0}(\alpha)$ such that the sequence

$$
l_{k}=\left(2^{-k n}\left(k+k_{0}\right)^{2}\right)^{1 /(n-\alpha p)}
$$

satisfies the condition $l_{k+1} \leq l_{k} / 2(k=0,1, \ldots)$. Moreover,

$$
\sum_{k=0}^{\infty} 2^{-k n} l_{k}^{\alpha p-n}<\infty
$$

Let $K_{\alpha}$ be the Cantor set corresponding to the sequence $\left\{l_{k}\right\}$, defined in [2, (5.3.1)]. Then $\left|K_{\alpha}\right|=0$ and by [2, Theorem 5.3.2], $\operatorname{cap}\left(K_{\alpha} ; B_{p}^{\alpha}\right)>0$. For $\lambda>0$, set

$$
K_{\alpha, \lambda}=\left\{x \in \mathbb{R}^{n}: \frac{x}{\lambda} \in K_{\alpha}\right\}
$$


There exists a function $f_{\alpha, \lambda} \in C_{0}^{\infty}$ such that $0 \leq f_{\alpha, \lambda}(x) \leq 1$ for all $x \in \mathbb{R}^{n}, f_{\alpha, \lambda}(x)=1$ in some neighborhood of $K_{\alpha, \lambda}$, and

$$
\left\|f_{\alpha, \lambda}\right\|_{b_{p}^{\alpha}}^{p} \leq \operatorname{cap}\left(K_{\alpha, \lambda} ; B_{p}^{\alpha}\right)+1 .
$$

Set $g_{\alpha, \lambda}(x)=f_{\alpha, \lambda}(\lambda x)$. Then $g_{\alpha, \lambda}(x)=1$ in some neighborhood of $K_{\alpha}$. Thus, using (4.14), we obtain

$$
\begin{aligned}
\operatorname{cap}\left(K_{\alpha} ; B_{p}^{\alpha}\right) & \leq\left\|g_{\alpha, \lambda}\right\|_{b_{p}^{\alpha}}^{p}=\lambda^{\alpha p-n}\left\|f_{\alpha, \lambda}\right\|_{b_{p}^{\alpha}}^{p} \\
& \leq \lambda^{\alpha p-n}\left(\operatorname{cap}\left(K_{\alpha, \lambda} ; B_{p}^{\alpha}\right)+1\right) .
\end{aligned}
$$

From here,

$$
\operatorname{cap}\left(K_{\alpha, \lambda} ; B_{p}^{\alpha}\right) \geq \lambda^{n-\alpha p} \operatorname{cap}\left(K_{\alpha} ; B_{p}^{\alpha}\right)-1
$$

Since $\operatorname{cap}\left(K_{\alpha} ; B_{p}^{\alpha}\right)>0$, we can choose such $\lambda(\alpha)>0$ that

$$
\alpha \operatorname{cap}\left(K_{\alpha, \lambda(\alpha)} ; B_{p}^{\alpha}\right)>1
$$

Thus, for any $0<\alpha<\min (1, n / p)$ there exists a compact set $E_{\alpha}$ such that

$$
\left|E_{\alpha}\right|=0 \quad \text { and } \quad \alpha \operatorname{cap}\left(E_{\alpha} ; B_{p}^{\alpha}\right)>1
$$

Let $j_{0}=\left[(\min (1, n / p))^{-1}\right]+1$. Set $E_{j}^{*}=E_{1 / j}, j \geq j_{0}$. Then

$$
\alpha \operatorname{cap}\left(E_{j}^{*} ; B_{p}^{\alpha}\right)>1 \quad \text { for } \quad \alpha=\frac{1}{j}\left(j \geq j_{0}\right) .
$$

Further, set $E=\cup_{j=j_{0}}^{\infty} E_{j}^{*}$. Then $|E|=0$. Let $0<\varepsilon<1$. There exists an open set $G$ such that $E \subset G$ and $|G|<\varepsilon$. We have

$$
\alpha \operatorname{cap}\left(G ; B_{p}^{\alpha}\right) \geq \alpha \operatorname{cap}\left(E_{j}^{*} ; B_{p}^{\alpha}\right)>1 \quad \text { for } \quad \alpha=\frac{1}{j} \quad\left(j \geq j_{0}\right) .
$$

Thus,

$$
\varlimsup_{\alpha \rightarrow 0} \alpha \operatorname{cap}\left(G ; B_{p}^{\alpha}\right) \geq 1,
$$

and equality (4.1) does not hold for the set $G$.

Remark 4.3. Our final remark concerns limiting relation (1.6). This relation was proved in [21] for the seminorm

$$
\left(\int_{\mathbb{R}^{n}} \int_{\mathbb{R}^{n}} \frac{|f(x+h)-f(x)|^{p}}{|h|^{n+\alpha p}} d x d h .\right)^{1 / p} .
$$

It is well known that this seminorm is equivalent to $\|f\|_{b_{p}^{\alpha}}$. We shall briefly discuss the limiting behaviour of $\alpha\|f\|_{b_{p}^{\alpha}}$. 
Assume that a function $f$ belongs to $B_{p, q}^{\alpha_{0}}\left(\mathbb{R}^{n}\right)$ for some $0<\alpha_{0}<1$. Then $f \in B_{p, q}^{\alpha}\left(\mathbb{R}^{n}\right)$ for any $0<\alpha \leq \alpha_{0}$. Moreover, it follows immediately from [10, Lemma 1] that

$$
\lim _{\alpha \rightarrow 0} \alpha^{1 / q}\|f\|_{b_{p, q}^{\alpha}}=q^{-1 / q} \sum_{j=1}^{n} \omega_{j}(f ;+\infty)_{p} .
$$

It is also easily seen that

$$
\lim _{h \rightarrow \infty} \int_{\mathbb{R}^{n}}\left|f\left(x+h e_{j}\right)-f(x)\right|^{p} d x=2\|f\|_{p}^{p} \quad(j=1, \ldots, n) .
$$

This equality and (2.5) imply that for a nonnegative $f$

$$
\omega_{j}(f ;+\infty)=2^{1 / p}\|f\|_{p} \quad(j=1, \ldots, n)
$$

and thus by (4.15)

$$
\lim _{\alpha \rightarrow 0} \alpha^{1 / q}\|f\|_{b_{p, q}^{\alpha}}=q^{-1 / q} 2^{1 / p} n\|f\|_{p} \quad \text { if } \quad f \geq 0 .
$$

However, equalities (4.16) and (4.17) fail to hold in a general case. We consider the following simple example for $n=1$. Let $I_{k}=[k, k+1)$ $(k=0,1, \ldots, 2 \nu)$. Set

$$
f_{\nu}(x)=\sum_{k=0}^{2 \nu}(-1)^{k} \chi_{I_{k}}(x) .
$$

Then $\left\|f_{\nu}\right\|_{p}=(2 \nu+1)^{1 / p}$. Further,

$$
\begin{aligned}
& \int_{\mathbb{R}}\left|f_{\nu}(x+1)-f_{\nu}(x)\right|^{p} d x \\
& \geq \sum_{k=0}^{2 \nu-1} \int_{I_{k}}\left|f_{\nu}(x+1)-f_{\nu}(x)\right|^{p} d x=2^{p+1} \nu .
\end{aligned}
$$

Thus,

$$
\omega\left(f_{\nu} ; 1\right)_{p} \geq 2\left(\frac{2 \nu}{2 \nu+1}\right)^{1 / p}\left\|f_{\nu}\right\|_{p} .
$$

It shows that the constant 2 on the right-hand side of (2.4) is optimal, and thus (4.16) and (4.17) may not be true.

\section{REFERENCES}

[1] D.R. Adams, The classification problem for the capacities associated with the Besov and Triebel-Lizorkin spaces, Banach Center Publ. 22 (1989), 9 - 24.

[2] D.R. Adams and L.I. Hedberg, Function Spaces and Potential Theory, Springer-Verlag Berlin Heidelberg New York 1999.

[3] C. Bennett and R. Sharpley, Interpolation of Operators, Academic Press, Boston 1988. 
[4] O.V. Besov, V.P. Il'in and S.M. Nikol'skiǔ, Integral Representations of Functions and Embedding Theorems, Vol. 1-2, Winston, Washington, D.C., Halsted Press, New York, $1978-1979$.

[5] J. Bourgain, H. Brezis and P. Mironescu, Another look at Sobolev spaces, Optimal Control and Partial Differential Equations. In honour of Professor Alain Bensoussan's 60th Birthday. J. L. Menaldi, E. Rofman, A. Sulem (eds), IOS Press, Amsterdam, 2001, 439 - 455.

[6] J. Bourgain, H. Brezis and P. Mironescu, Limiting Embedding Theorems for $W^{s, p}$ when $s \uparrow 1$ and applications, J. D'Analyse Math. 87 (2002), 77 - 101.

[7] H. Brezis, How to recognize constant functions. Connections with Sobolev spaces, (Russian) Uspekhi Mat. Nauk 57 (2002), no. 4(346), 59-74; English transl. in Russian Math. Surveys 57 (2002), no. 4, 693-708.

[8] H. Brezis, Functional Analysis, Sobolev Spaces and Partial Differential Equations, Springer New York Doldrecht Heidelberg London 2011.

[9] A.M. Caetano, A. Gogatishvili, B. Opic, Embeddings and the growth envelope of Besov spaces involving only slowly varying smoothness, J. Approx. Theory, 163 (2011), 1373 - 1399.

[10] G.E. Karadzhov, M. Milman and J. Xiao, Limits of higher order Besov spaces and sharp reiteration theorems, J. Funct. Anal. 221 (2005), 323 - 339.

[11] V.I. Kolyada, On imbedding in classes $\varphi(L)$, Izv. Akad. Nauk SSSR Ser. Mat. 39 (1975), 418 - 437; English transl.: Math. USSR Izv. 9 (1975), 395 - 413.

[12] V.I. Kolyada, Estimates of rearrangements and embedding theorems, Mat. Sb. 136 (1988), 3 - 23; English transl.: Math. USSR-Sb. 64 (1989), no. 1, 1 - 21.

[13] V.I. Kolyada, Rearrangements of functions and embedding theorems, Uspekhi matem. nauk 44 (1989), no. 5, 61 - 95; English transl. in Russian Math. Surveys 44 (1989), no. 5, $73-118$.

[14] V.I. Kolyada, Rearrangement of functions and embedding of anisotropic spaces of Sobolev type, East J. Approx. 4 (1998), no. 2, 111 - 199.

[15] V.I. Kolyada, Mixed norms and Sobolev type inequalities, Banach Center Publ. 72 (2006), $141-160$.

[16] V.I. Kolyada, On embedding theorems, in: Nonlinear Analysis, Function Spaces and Applications, vol. 8 (Proceedings of the Spring School held in Prague, 2006), Prague, 2007, 35 - 94.

[17] V.I. Kolyada and A.K. Lerner, On limiting embeddings of Besov spaces, Studia Math. 171, no. 1 (2005), 1 - 13.

[18] G. Leoni, A First Course in Sobolev Spaces, Graduate Studies in Mathematics, v. $105,2009$.

[19] G. Leoni, D. Spector, Characterization of Sobolev and BV spaces, J. Funct. Anal. 261 (2011), 2926 - 2958.

[20] V. Maz'ya, Sobolev spaces with applications to elliptic partial differential equations, Second, revised and augmented edition. Grundlehren der Mathematischen Wissenschaften, 342. Springer, Heidelberg, 2011.

[21] V. Maz'ya and T. Shaposhnikova, On the Bourgain, Brezis, and Mironescu theorem concerning limiting embeddings of fractional Sobolev spaces, J. Funct. Anal. 195 (2002), no. 2, 230 - 238.

[22] M. Milman, Notes on limits of Sobolev spaces and the continuity of interpolation scales, Trans. Amer. Math. Soc. 357 (2005), no. 9, 3425 - 3442. 
[23] S.M. Nikol'skiü, Approximation of Functions of Several Variables and Imbedding Theorems, Springer - Verlag, Berlin - Heidelberg - New York, 1975.

[24] E.M. Stein, Singular Integrals and Differentiability Properties of Functions, Princeton Univ. Press, 1970.

[25] H. Triebel, Limits of Besov norms, Arch. Math. 96 (2011), 169 - 175.

[26] P.L. Ul'yanov, Embedding of certain function classes $H_{p}^{\omega}$, Izv. Akad. Nauk SSSR Ser. Mat. 32 (1968), 649 - 686; English transl. in Math. USSR Izv. 2 (1968), $601-637$.

Department of Mathematics, Karlstad University, Universitetsgatan 1, 65188 KARLSTAD, SWEDEN

E-mail address: viktor.kolyada@kau.se 